\title{
Improved results of induction chemoradiation before surgical intervention for selected patients with stage IIIA-N2 non-small cell lung cancer
}

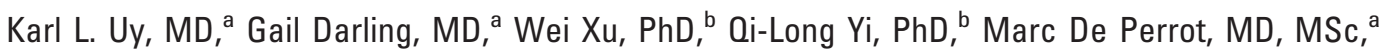
Andrew F. Pierre, MD, MSc, ${ }^{a}$ Thomas K. Waddell, MD, MSc, PhD, ${ }^{a}$ Michael R. Johnston, MD, Andrea Bezjak, MD, MSc, ${ }^{c}$ Frances A. Shepherd, MD, ${ }^{d}$ and Shaf Keshavjee, MD, MSc ${ }^{a}$

Earn CME credits at http://cme.ctsnetjournals.org
From the Division of Thoracic Surgery, ${ }^{\text {a }}$ the Department of Biostatistics, ${ }^{\mathrm{b}}$ the Department of Radiation Oncology, ${ }^{\mathrm{c}}$ and the Department of Medical Oncology and Hematology, ${ }^{\mathrm{d}}$ University Health Network, University of Toronto, Toronto, Ontario, Canada.

Read at the 11th World Conference on Lung Cancer, Barcelona, Spain, July 3-6, 2005.

Received for publication Aug 1, 2006; revisions received Jan 4, 2007; accepted for publication Jan 18, 2007.

Address for reprints: Gail Darling, MD, Division of Thoracic Surgery, 9N-955 Toronto General Hospital, 200 Elizabeth St, Toronto, Ontario M5G2C4, Canada (Email: gail.darling@uhn.on.ca).

J Thorac Cardiovasc Surg 2007;134:188-93

$0022-5223 / \$ 32.00$

Copyright () 2007 by The American Association for Thoracic Surgery

doi:10.1016/j.jtcvs.2007.01.078
Objective: Optimal management of stage IIIA-N2 non-small cell lung cancer remains controversial. The surgical arm of the North American Intergroup 0139 trial was adopted as the standard treatment for patients with resectable N2 disease at the University Health Network. Results after 7 years of experience are reported.

Methods: This is a retrospective study of consecutive patients with biopsy-proved T1-3 N2 M0 lung cancer who underwent induction chemoradiation before surgical intervention from January 1997 through August 2004. Induction chemotherapy consisted of cisplatin, $50 \mathrm{mg} / \mathrm{m}^{2}$, on days 1 and 8 ; etoposide, $50 \mathrm{mg} / \mathrm{m}^{2}$, on days 1 to 5, weeks 1 and 5; and concurrent daily external beam radiotherapy to $45 \mathrm{~Gy}$. Lung resection was performed within 6 weeks of completion of chemoradiation, followed by 2 further cycles of consolidation chemotherapy.

Results: Between January 1997 and August 2004, 40 patients were treated according to this protocol $(25 \% \mathrm{~T} 1,62.5 \% \mathrm{~T} 2,7.5 \% \mathrm{~T} 3$, and $5 \% \mathrm{~T} 4)$. Overall and disease-free median survivals were 40 and 37.1 months, respectively, whereas overall and disease-free 3 -year survivals were $51.7 \%$ and $52.3 \%$, respectively. R0 resection was achieved in $92.5 \%$. The overall operative mortality rate was $7.5 \%$ ( $0 \%$ for lobectomy and $27 \%$ for pneumonectomy). Notably, all mortalities occurred within the first 2 years of our experience with this regimen.

Conclusion: Chemoradiation before pulmonary resection in carefully selected patients with surgically resectable stage IIIA (N2) non-small cell lung cancer can lead to improved overall and disease-free survival.

$\mathrm{T}$ The burden of disease in stage IIIA (N2) non-small cell lung cancer (NSCLC) varies from microscopic, single-station, mediastinal nodal involvement to bulky, multistation, fixed, mediastinal nodal disease. Although the overall prognosis for stage IIIA disease is poor, the prognosis varies based on the extent of N2 disease. Controversy regarding optimal management continues 12 years after Martini and colleagues' ${ }^{1}$ landmark article reporting the benefit of preoperative chemotherapy for such patients. Subsequent randomized trials have reported conflicting results. ${ }^{2-5}$ The results of the recently completed but as yet unpublished North American Intergroup 0139 randomized trial might clarify whether induction chemoradiation followed by surgical resection has advantages over definitive chemoradiation.

An interim analysis of the Intergroup 0139 trial $^{6}$ demonstrated that induction chemoradiation followed by surgical resection provided better progression-free survival but not overall survival. The excess mortality experienced by patients who required pneumonectomy after induction chemoradiotherapy adversely affected the overall survival in the surgical group. The $26 \%$ operative mortality rate in patients 


\section{Abbreviations and Acronyms}

NSCLC $=$ non-small cell lung cancer

undergoing pneumonectomy (29\% mortality for complex and $21.7 \%$ for simple pneumonectomies) was unexpected from the phase II trials and is considered excessive in the modern era. After closure of the 0139 trial, the surgical arm of the protocol was adopted for all patients with resectable stage IIIA-N2 disease at the Toronto General Hospital, University Health Network.

\section{Materials and Methods}

This is a retrospective series of patients with biopsy-proved N2 disease who underwent surgical resection after induction chemoradiotherapy in our institution. During the time period of the study, there were 550 patients treated at our cancer center with N2 disease. Of this group, 50 patients were treated with definitive chemoradiotherapy (including 20 patients treated on the nonsurgical arm of Intergroup 0139), and 40 patients were treated with induction chemoradiotherapy followed by surgical resection (including 16 patients treated on the surgical arm of Intergroup study 0139). The remainder of the patients (460) did not receive or complete either of these treatment plans because of poor perfor-

TABLE 1. Patient characteristics and outcomes

\begin{tabular}{|c|c|c|c|c|c|}
\hline \multirow[b]{2}{*}{ Characteristics } & \multirow[b]{2}{*}{$\mathbf{N}(\%)$} & \multicolumn{2}{|c|}{$\begin{array}{l}\text { Median survival } \\
\text { (mo) }\end{array}$} & \multicolumn{2}{|c|}{$P$ value } \\
\hline & & os & DFS & OS & DFS \\
\hline All patients & 40 & 40 & 37.1 & & \\
\hline Sex & & & & .25 & .28 \\
\hline Male & $20(50)$ & 40 & 37.1 & & \\
\hline Female & $20(50)$ & 27.3 & 26.3 & & \\
\hline $\begin{array}{l}\text { CT-detected N2 (>1 } \\
\mathrm{cm})\end{array}$ & & & & .64 & .53 \\
\hline Yes & $29(72.5)$ & 41.7 & 37.1 & & \\
\hline No & $11(27.5)$ & 22.3 & 20.9 & & \\
\hline $\begin{array}{l}\text { Involved nodes at } \\
\text { mediastinoscopy }\end{array}$ & & & & .11 & .36 \\
\hline Single & $26(65)$ & NAt & 37.1 & & \\
\hline Multiple & $14(35)$ & 40 & 28.3 & & \\
\hline cT status* & & & & .009 & .12 \\
\hline 1 & $10(25)$ & NA & NA & & \\
\hline 2 & $25(62.5)$ & 40 & 26.3 & & \\
\hline 3 & $3(7.5)$ & 9.6 & NA & & \\
\hline 4 & $2(5)$ & NA & 22.4 & & \\
\hline Pathologic Response* & & & & .006 & .0006 \\
\hline None & $13(32.5)$ & 21.1 & 18.4 & & \\
\hline$C R+P R$ & $27(67.5)$ & NAt & 48.5 & & \\
\hline
\end{tabular}

$O S$, Overall survival; $D F S$, disease-free survival; $C T$, computed tomography; $N A$, not applicable; $C R$, complete pathological response; $P R$, partial pathologic response. *Statistically significant difference. †Median survival not yet reached.
TABLE 2. Morbidity and mortality by type of resection

\begin{tabular}{llll}
\hline Type of resection & $\mathbf{N}$ & $\begin{array}{c}\text { Morbidity } \\
\mathbf{N}(\%)\end{array}$ & $\begin{array}{c}\text { Mortality } \\
\mathbf{N}(\%)\end{array}$ \\
\hline $\begin{array}{l}\text { Lobectomy } \\
\text { Pneumonectomy }\end{array}$ & 29 & $2(6.9)$ & 0 \\
$\quad 11$ & $4(36)$ & $3(27)$ \\
$\quad$ Right & & & \\
$\quad$ Simple & 2 & 0 & 0 \\
$\quad$ Complex & 4 & $3(75)$ & $2(50)$ \\
$\quad$ Left & 2 & 0 & 0 \\
Simple & 3 & $1(33)$ & $1(33)$ \\
$\quad$ Complex & 4 & 0 & 0 \\
$\quad$ All simple & 7 & $4(57)$ & $3(43)$ \\
$\quad$ All complex & 40 & $6(15)$ & $3(7.5)$ \\
Overall & & & \\
\hline
\end{tabular}

mance status, medical comorbidities, or progression on treatment or because they had already had part of their treatment (including surgical intervention) before referral to our institution.

Involvement of $\mathrm{N} 2$ nodes was confirmed by means of mediastinoscopy in all cases. Patients with T1 to T3 tumors were included, as well as those with T4 tumors if the T4 designation was due to satellite nodules but not other T4 categories. All patients were classified as having Eastern Cooperative Oncology Group performance status of 0 to 1 before induction treatment and proceeded to surgical resection if there was no evidence of tumor progression on postinduction restaging with computed tomographic scans of the chest and abdomen, bone scans, and brain magnetic resonance imaging.

Induction treatment consisted of 2 cycles of cisplatin, $50 \mathrm{mg} /$ $\mathrm{m}^{2}$, on days 1 and 8 and etoposide, $50 \mathrm{mg} / \mathrm{m}^{2}$, on days 1 to 5 , with concurrent external beam radiation of 1.8 Gy per day beginning on day 1 to a total dose of 45 Gy in 25 doses. All patients completed the induction regimen. Lobectomy or pneumonectomy with complete mediastinal lymph node dissection was performed within 6 weeks of completion of the induction regimen. Two additional cycles of consolidation chemotherapy were recommended postoperatively with the same drug combination.

A complex pneumonectomy was defined in this series as a pneumonectomy requiring intrapericardial dissection because of bulky central disease. Disease-free survival (freedom from death and recurrence) and overall survival were calculated by applying the Kaplan-Meier method starting at the date of the first chemotherapy treatment; the log-rank test was used to compare survival curves. The statistical analysis was performed with version 9.1 of the SAS System and User's Guide (SAS Institute, Cary, NC).

\section{Results}

Forty patients (20 male patients; mean age, $59.9 \pm 8.8$ years) completed the induction regimen between January 1997 and August 2004. All patients who survived surgical intervention (37) received one cycle of postoperative chemotherapy, and 35 received both cycles. Median follow-up was 22 months. The majority of tumors were T2 (62.5\%). Enlarged N2 nodes $(>1 \mathrm{~cm})$ were detected by means of computed tomography at a single station only in $65 \%$ of 


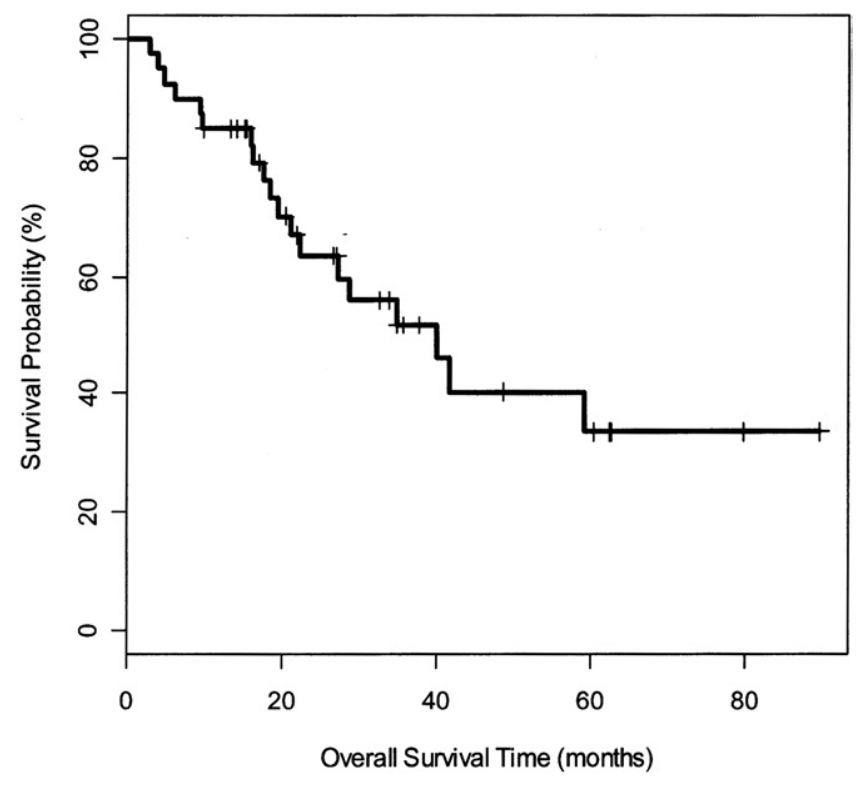

Figure 1. Overall survival.

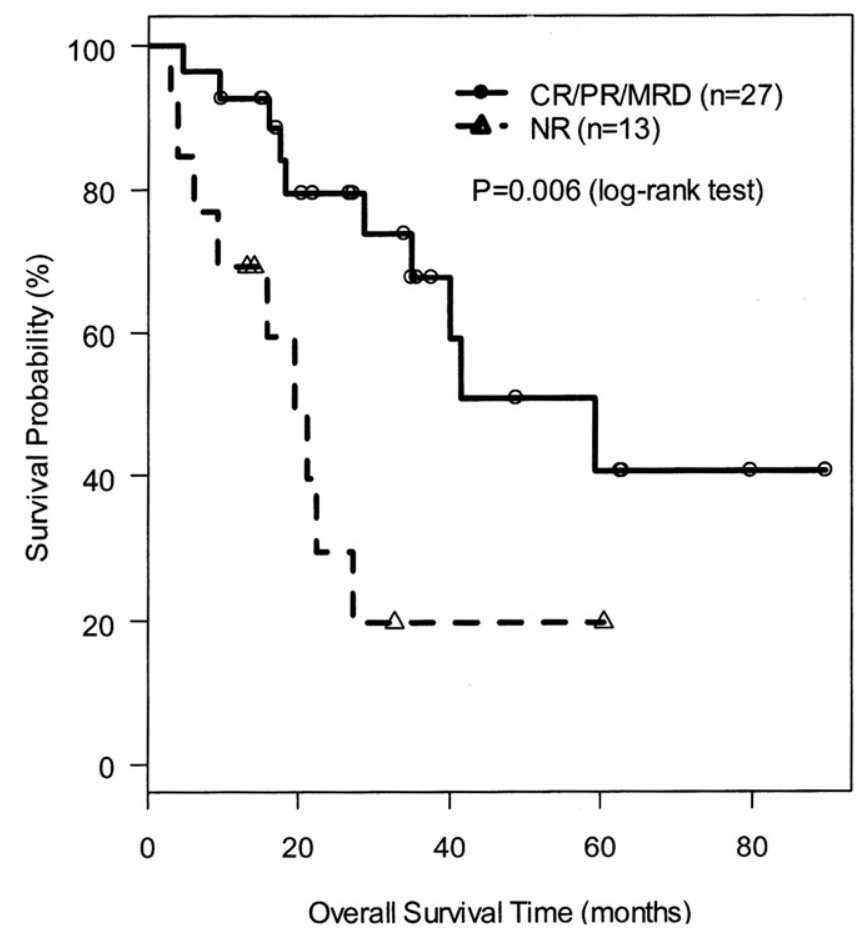

Figure 3. Overall survival by pathologic response. CR, Complete pathological response; $P R$, partial pathological response; $M R D$, minimum residual disease defined as less than $10 \%$ viable tumor cells; $N R$, no response. patients (Table 1). Two patients with T4 satellite nodules were included in the analysis.

Overall and disease-free median survivals were 40 and 37.1 months, and overall and disease-free 3-year survivals were $51.7 \%$ and $52.3 \%$, respectively (Figure 1). Overall

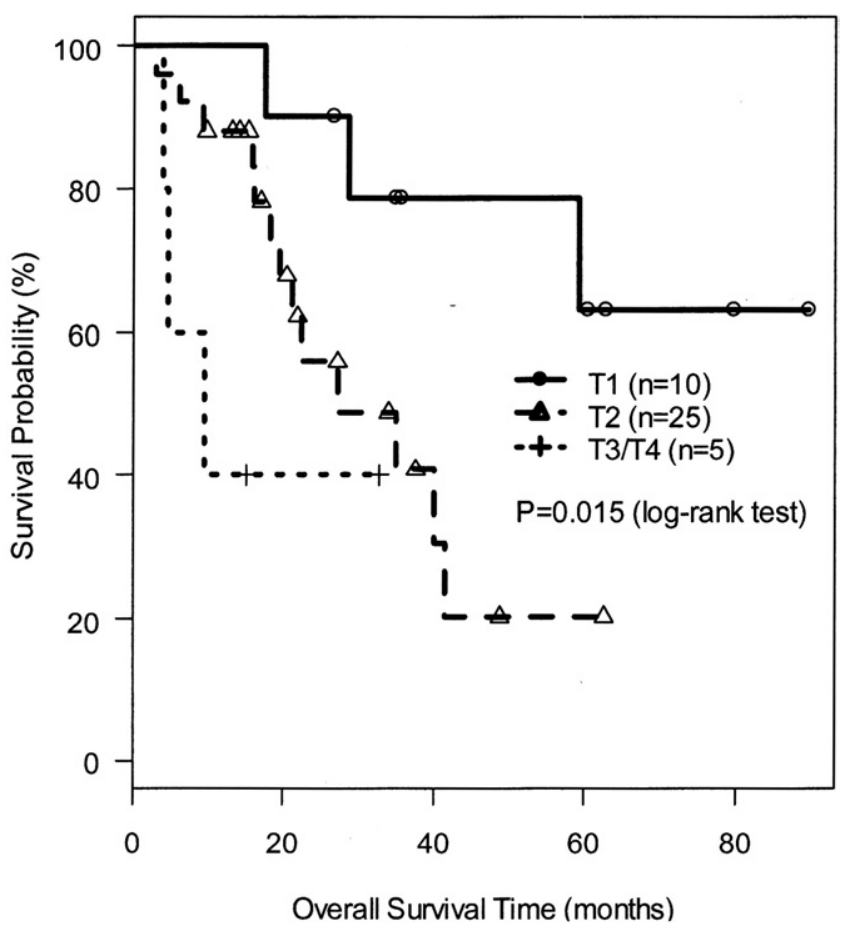

Figure 2. Overall survival by $\mathrm{T}$ stage. survival was better with lower T stages $(P<.015$, Figure 2 ), and there was a trend toward improved disease-free survival. There was a trend toward better overall survival in patients with single-station involvement, but this was not statistically significant (Figure 3). There was no significant difference in survival between patients with enlarged nodes on computed tomographic scans versus those with normalsized nodes.

Pathologic response was categorized as no response versus any response. Partial pathologic response or complete pathologic response was found in $20(50 \%)$ and $7(17.5 \%)$ patients, respectively, for an overall response rate of $67.5 \%$ (27 patients), and this translated into a significant overall (Figure 4) and disease-free survival advantage. Resection was accomplished in all patients by means of lobectomy in 29 and pneumonectomy in 11, with an overall operative mortality rate of $7.5 \%$ and morbidity rate of $15 \%$ (Table 2). There was no long-term survival difference between the 2 types of resections; however, operative mortality was $0 \%$ for patients undergoing lobectomy, with a $6.9 \%$ morbidity rate. There were few pneumonectomies, but these were associated with a $27 \%$ operative mortality and a $36 \%$ morbidity. There was no mortality after 4 simple pneumonectomies, but there were 3 deaths in 7 complex pneumonec- 


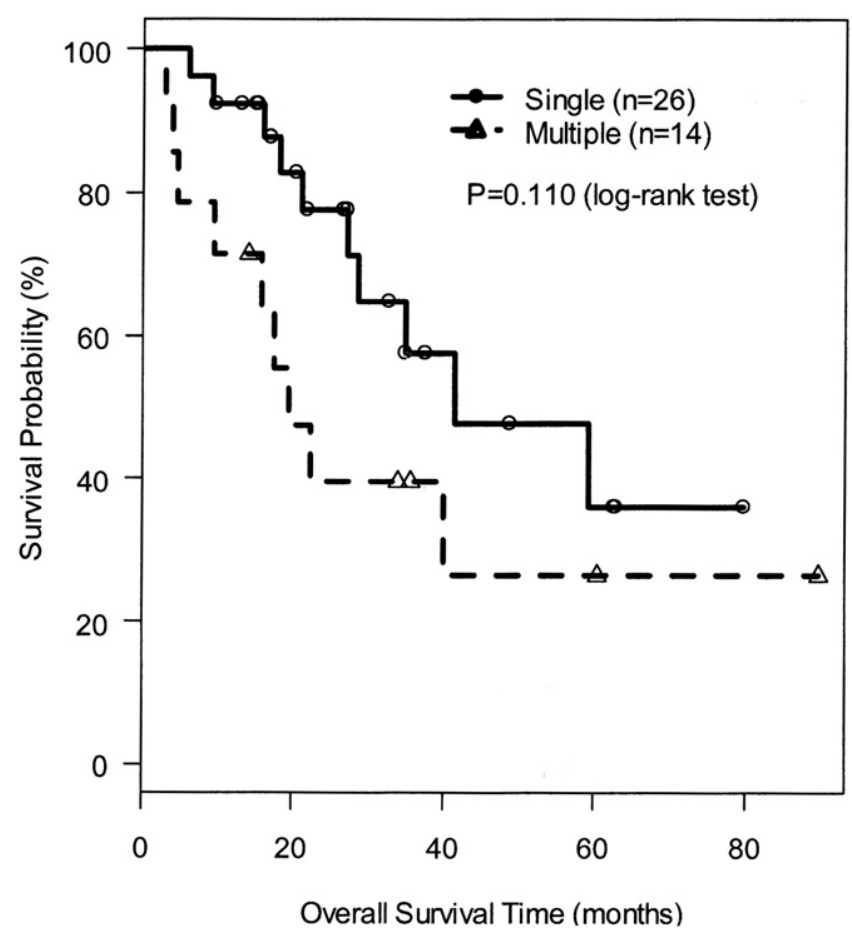

Figure 4. Overall survival by number of involved nodal stations determined at mediastinoscopy.

tomies (43\%), all of which occurred early (before 1999) in our experience. Right pneumonectomy was associated with a higher mortality (2/6 [33\%]) versus left pneumonectomy $(1 / 5[20 \%])$. Death was due to adult respiratory distress syndrome in 2 patients and postoperative hemorrhage in 1 patient. Major morbidities included 2 instances of massive intraoperative hemorrhage from dissection of centrally located tumors, one of which required cardiopulmonary bypass, and 1 each of cardiac tamponade and pulmonary embolism. Importantly, there was no mortality in the 34 consecutive lung resections over the last 5 years of the study, including 5 patients who required pneumonectomy. R0 resection was accomplished in 37 patients, with only 2 $\mathrm{R} 1$ and $1 \mathrm{R} 2$ resections. The site of relapse was distant in $89 \%$, with the brain being the most common site of distant metastasis in $62.5 \%$ of relapsing patients.

\section{Discussion}

The optimum treatment strategy for stage IIIA-N2 NSCLC remains controversial. Although some patients are cured with surgical intervention alone, results vary depending on the extent of nodal disease (29\%-35\%). ${ }^{7,8}$ Two small randomized trials suggest that surgical intervention alone is inferior to preoperative chemotherapy followed by surgical resection for patients with clinically evident N2 disease, ${ }^{9,10}$ whereas a third trial showed no difference. ${ }^{11}$ In all 3 trials some patients received postoperative radiotherapy. Other trials have suggested that adjuvant chemotherapy after surgical resection might improve survival over surgical intervention alone. ${ }^{12,13}$ On the basis of a number of phase II and small phase III trials, neoadjuvant chemotherapy followed by surgical resection has been widely practiced. The North American Intergroup 0139 multi-institutional randomized controlled trial addressed the question of definitive chemoradiation versus induction chemoradiation followed by surgical resection for patients with stage IIIA-N2 NSCLC. The mature results are awaited; however, the initial results of this trial showed there was an increase in disease-free survival in the surgical arm, but there was no difference in overall survival between the 2 treatment arms. The lack of difference in overall survival was attributed to early deaths in the surgical arm attributable mostly to high operative mortality after pneumonectomy $(26 \%){ }^{6}$ On the basis of these preliminary results, many centers have avoided surgical therapy in all patients with N2 disease and adopted chemoradiotherapy alone as the standard treatment for stage IIIA-N2 NSCLC.

At the Toronto General Hospital, the surgical arm of the Intergroup 0139 trial protocol was continued as the standard treatment for selected fit patients with resectable stage IIIA-N2 NSCLC after closure of accrual to the trial. After the initial presentation of the 0139 results, our impression was that the improved disease-free interval in the surgical arm might indeed be clinically important, and therefore we reviewed our own center's experience.

Our single-center experience with induction concurrent chemoradiotherapy with cisplatin and etoposide with $45 \mathrm{~Gy}$ of radiation demonstrates a median disease-free survival of 37 months and a median overall survival of 40 months. In the surgical arm of the 0139 trial, median disease-free survival was 13.4 months, and median overall survival was 22 months. The 3-year disease-free survival and overall 3 -year survival in our series were $52 \%$ and $52 \%$, respectively, versus $37 \%$ and $28 \%$ in the surgical arm of the 0139 trial. We recognize that although a number of surgeons over time were involved, our results still represent a retrospective study of a single-institution experience and therefore are not directly comparable with the results of a prospective multicenter randomized trial. It is also important to note that our results are not based on an intention-to-treat analysis but rather on a review of those selected patients who were able to complete this treatment regimen and hence is inherently biased. However, we believe that this is important information in that it documents that there is a population of patients with N2 NSCLC who are candidates for potentially curative surgical therapy. Moreover, careful analysis of such patients from a single institution might help to elucidate important factors that ultimately might be applied to improve patient 
selection outcomes of lung cancer treatment in this challenging group of patients.

Similar to the 0139 trial, however, we found that there was indeed significantly higher mortality in the subgroup of patients who required a complex pneumonectomy to accomplish their resection, although only in this small subgroup. Furthermore, all deaths occurred early in our pre-1999 experience during accrual to the 0139 trial and none during the post-0139 experience. In the lobectomy $(\mathrm{n}=29)$ and simple pneumonectomy $(\mathrm{n}=4)$ subgroups, which comprised $77.5 \%$ of patients, mortality was $0 \%$. Despite a $43 \%$ operative mortality rate for the total of 7 patients who underwent complex pneumonectomy, the median overall survival of the entire group (including all patients undergoing resection) was 40 months, and 3-year survival was $51.7 \%$, which compares favorably with the results of older published series. ${ }^{2,3,14,15}$

We attempted to identify the key factors that contributed to the high mortality rate of complex pneumonectomy and found that all 3 mortalities occurred within the first 2 years of our institution's experience with neoadjuvant chemoradiation. Of the 3 mortalities, all patients had bulky central tumors with challenging operations. The cause of death was adult respiratory distress syndrome in 2 and hemorrhage in the third. The subsequent 34 patients (including 2 additional patients who underwent complex pneumonectomies) and 4 who required only simple pneumonectomies did not experience a single mortality.

The hazards of performing a lung resection after induction chemotherapy with or without radiation have been investigated, with variable results reported; however, the larger series have consistently reported higher morbidity and mortality rates after induction compared with standard lung resections. Overall reported mortality rates for lung resections for primary lung cancer without induction are $1.3 \%$ to $3.7 \%,{ }^{16-18}$ with rates for lobectomy of $1.2 \%$ to $2.9 \%$ and $3.2 \%$ to $6.2 \%$ for pneumonectomy, whereas the larger series of postinduction lung resections report operative mortalities of $2.4 \%$ to $3.8 \%$ for lobectomy and $7.2 \%$ to $12 \%$ for pneumonectomy. ${ }^{18-20}$

Exploratory analysis of the Intergroup 0139 data reporting a subset analysis of patients demonstrated that there was a significant survival advantage for patients who underwent lobectomy compared with a matched group who underwent definitive chemoradiation (34 vs 22 months' median survival). There was no difference in survival, however, when the pneumonectomy group was compared with a matched chemoradiation group. ${ }^{6}$ This trial had a $21.7 \%$ operative mortality rate for simple pneumonectomy, as opposed to our current experience of $0 \%$ mortality. The smoother postoperative courses of our current-era patients undergoing pneumonectomy leads us to believe that the operative mortality rate for this subgroup will continue to be acceptably low and that, similar to the lobectomy subgroup, they should be selectively considered for a strategy of neoadjuvant chemoradiation.

In our review we found that, over time, surgeons selected fewer patients with bulky central tumors or with bulky multistation disease, in which resection might have been predicted to be difficult. However, there was no change in the number of patients with single-station disease. From a physiologic perspective, we did not observe any changes over time in pulmonary function testing. Our results suggest that with increased experience and refined patient selection, treatment according to the surgical arm of the 0139 trial is safe and might lead to improved survival in patients with IIIA-N2 lung cancer compared with historical reports or the Intergroup 0139 trial. On the basis of our results, we believe that carefully selected patients with stage IIIA-N2 NSCLC should be offered neoadjuvant chemoradiotherapy followed by surgical resection. We believe that better patient selection, operative technique, and increased experience operating after chemoradiotherapy contributed to this improvement in results.

In many centers, definitive chemoradiotherapy has become the recommended treatment in patients who require pneumonectomy because of the high operative mortality reported in the Intergroup 0139 trial. The recently presented European Organization for Research and Treatment of Cancer 08941 trial reports equivalent survival for patients treated with chemotherapy followed by either radiation or surgical intervention. ${ }^{21}$ This trial, however, specifically included patients with stage IIIA disease "in whom the nodal disease (N2) was considered irresectable" by the thoracic surgeon at the time of initial assessment. We concur that such patients are likely best managed with definitive chemoradiotherapy, whereas for those patients in whom the nodal disease and primary tumor are technically resectable, surgical intervention should be considered as part of the treatment algorithm.

\section{Conclusions}

On the basis of our results, patients at our center with stage IIIA-N2 NSCLC whose tumors are amenable to a lobectomy or pneumonectomy are offered neoadjuvant chemoradiation before surgical resection as the treatment of choice. Clearly, this treatment strategy is not accepted universally, and the role of surgical intervention in stage III N2 NSCLC continues to be debated. We believe that highly selected patients in whom disease is considered technically resectable (particularly those with microscopic N2 disease) do have a chance to achieve improved long-term survival with trimodality therapy. As with any complex therapy, continued experience with careful analysis and reporting of results will allow us to more clearly define the patients who are 
most likely to derive benefit from multimodality treatment for stage IIIA-N2 lung cancer.

\section{References}

1. Martini N, Kris MG, Flehinger BJ, et al. Preoperative chemotherapy for stage IIIa (N2) lung cancer: the Sloan-Kettering experience with 136 patients. Ann Thorac Surg. 1993;55:1365-74.

2. Roth JA, Atkinson EN, Fossella F, et al. Long-term follow-up of patients enrolled in a randomized trial comparing perioperative chemotherapy and surgery with surgery alone in resectable stage IIIA non-small-cell lung cancer. Lung Cancer. 1998;21:1-6.

3. Rosell R, Gomez-Codina J, Camps C, et al. Preresectional chemotherapy in stage IIIA non-small cell lung cancer: a 7-year assessment of a randomized controlled trial. Lung Cancer. 1999;26:7-14.

4. Depierre A, Milleron B, Moro-Sibilot D, et al. Preoperative chemotherapy followed by surgery compared with primary surgery in resectable stage I (except T1N0), II, and IIIa non-small-cell lung cancer. $J$ Clin Oncol. 2002;20:247-53.

5. Johnstone DW, Byhardt RW, Ettinger D, Scott CB. Phase III study comparing chemotherapy and radiotherapy with preoperative chemotherapy and surgical resection in patients with non-small-cell lung cancer with spread to mediastinal lymph nodes (N2); final report of RTOG 89-01. Int J Radiat Oncol Biol Phys. 2002;54:365-9.

6. Albain KS, Swann RS, Rusch VR, et al. Phase III study of concurrent chemotherapy and radiotherapy (CT/RT) vs CT/RT followed by surgical resection for stage IIIA(pN2) non-small cell lung cancer (NSCLC): outcomes update of North American Intergroup 0139 (RTOG 9309). Proc Am Soc Clin Oncol. 2005; abstract 7014.

7. Daly BD, Mueller JD, Faling LJ, et al. N2 lung cancer: outcome in patients with false negative computed tomographic scans of the chest. J Thorac Cardiovasc Surg. 1993;105:904-10.

8. Keller SM, Vangel MG, Wagner $\mathrm{H}$, et al. Prolonged survival in patients with resected non-small cell lung cancer and single-level N2 disease. J Thorac Cardiovasc Surg. 2004;128:130-7.

9. Roth JA, Fossella F, Komaki R, et al. A randomized trial comparing perioperative chemotherapy and surgery with surgery alone in resectable stage IIIA nonsmall cell lung cancer. J Natl Cancer Inst. 1994; 86:673-80.

10. Rosell R, Gomez-Codina J, Camps C, et al. A randomized trail comparing preoperative chemotherapy plus surgery with surgery alone in patients with nonsmall cell lung cancer. $N$ Engl $J$ Med. 1994;330:153-8.

11. Depierre A, Milleron B, Moro-Sibilot D, et al. Preoperative chemotherapy followed by surgery compared with primary surgery in resectable stage I ( except T1N00, II, and IIIA nonsmall cell lung cancer. $J$ Clin Oncol. 2002;20:247-53.

12. The International Association for Lung Cancer Trial Collaborative Group. Cisplatin-based adjuvant chemotherapy in patients with completely resected non-small-cell lung cancer. N Engl J Med. 2004;350: 351-60.

13. Douillard JY, Rosell R, De Lena M, et al. ANITA:phase III adjuvant vinorelbine and cisplatin versus observation in completely resected non-small-cell lung cancer patients: final results after 70-month median follow-up. Lancet Oncol. 2006;7:719-27.

14. Voltolini L, Luzzi L, Ghiribelli C, Paladini P, Di Bisceglie M, Gotti G. Results of induction chemotherapy followed by surgical resection in patients with stage IIIa (N2) non-small cell lung cancer: the importance of the nodal down-staging after chemotherapy. Eur J Cardiothorac Surg. 2001;20:1106-12.

15. Wada H, Nakamura T, Nakamoto K, Maeda M, Watanabe Y. Thirtyday operative mortality for thoracotomy in lung cancer. $J$ Thorac Cardiovasc Surg. 1998;115:70-3.

16. Fang D, Zhang D, Huang G, Zhang R, Wang L, Zhang D. Results of surgical resection of patients with primary lung cancer: a retrospective analysis of 1,905 cases. Ann Thorac Surg. 2001;72:1155-9.

17. Ginsberg RJ, Hill LD, Eagan RT, et al. Modern thirty-day operative mortality for surgical resections in lung cancer. $J$ Thorac Cardiovasc Surg. 1983;86:654-8.

18. Stamatis G, Djuric D, Everhardt W, et al. Postoperative morbidity and mortality after induction chemoradiotherapy for locally advanced lung cancer: an analysis of 350 operated patients. Eur J Cardiothorac Surg. 2002;22:292-7.

19. Martin J, Ginsberg RJ, Abolhoda A, et al. Morbidity and mortality after neoadjuvant therapy for lung cancer: the risks of right pneumonectomy. Ann Thorac Surg. 2001;72:1149-54.

20. Doddoli C, Barlesi F, Trousse D, et al. One hundred consecutive pneumonectomies after induction therapy for non-small cell lung cancer: an uncertain balance between risks and benefits. $J$ Thorac Cardiovasc Surg. 2005;130:416-25.

21. Van Schil P, Van Meerbeeck J, Kramer G, et al. Morbidity and mortality in the surgery arm of EORTC 08941 trial. Eur Respir J. 2005;26:192-7. 